\title{
La función pulmonar no está pasada de moda
}

\author{
Lung Function is Not Outdated \\ Juana Martínez Llorens ${ }^{\mathrm{a}, \mathrm{b}, \mathrm{h}, *}$, Felip Burgos ${ }^{\mathrm{c}, \mathrm{d}, \mathrm{e}, \mathrm{h}}$ y Juan B. Galdiz ${ }^{\mathrm{f}, \mathrm{g}, \mathrm{h}}$ \\ a Servicio de Neumología, Hospital del Mar-IMIM, Parc de Salut Mar, Barcelona, España, Barcelona, España \\ ${ }^{\mathrm{b}}$ DCEXS, Universitat Pompeu Fabra, Barcelona, España \\ c Centro de Diagnóstico Respiratorio, Hospital Clínic, Barcelona, España \\ d Institut d'Investigacions Biomèdiques August Pi i Sunyer (IDIBAPS), Barcelona, España \\ e Universitat de Barcelona, Barcelona, España \\ ${ }^{\mathrm{f}}$ Laboratorio de Exploración Funcional, Servicio Neumología, Hospital Universitario Cruces, Bilbao, España \\ ${ }^{g}$ Biocruces, Bilbao, España \\ ${ }^{\mathrm{h}}$ Centro de Investigación en Red de Enfermedades Respiratorias (CIBERES)-Madrid CIBERES, ISCiii, Madrid, España
}

La valoración de la función pulmonar parece no atraer a los profesionales sanitarios que atienden a los pacientes con enfermedades respiratorias como antaño. Sin embargo, las pruebas de función pulmonar (PFP) son cada vez más importantes para el diagnóstico y el tratamiento de muchas enfermedades pulmonares. Así, en el caso de la fibrosis pulmonar idiopática, se han desarrollado últimamente 2 tratamientos antifibróticos, la pirferidona y el nintedanib, cuya eficacia se ha valorado en relación con el enlentecimiento en la caída de la capacidad vital forzada ${ }^{1}$. Además de la espirometría, otros parámetros de función pulmonar, como es el factor de transferencia del monóxido de carbono (TLCo), se consideran fundamentales para valorar la eficacia de otros nuevos fármacos antifibróticos ${ }^{1}$. En la enfermedad pulmonar obstructiva crónica, la espirometría forzada es actualmente la prueba necesaria para su diagnóstico y estadificación ${ }^{2}$. Sin embargo, cada vez son más los autores que consideran que otros parámetros de las PFP, como TLco o la oscilometría forzada (FOT), son fundamentales para un diagnóstico precoz y que, por tanto, deben correlacionarse con los síntomas y además permitir valorar la eficacia de nuevos tratamientos para estos pacientes ${ }^{3}$. Lo mismo se puede extrapolar a otras enfermedades tan prevalentes como el asma bronquial o las enfermedades neruromusculares. Asimismo, se ha demostrado que los sujetos con un desarrollo inadecuado de los pulmones presentan parámetros de función pulmonar anormales en su desarrollo, lo que conlleva una mayor prevalencia de enfermedades cardiovasculares y metabólicas ${ }^{4,5}$ y un mayor riesgo de mortalidad por enfermedad cardiaca y pulmonar ${ }^{4}$.

Otra de las peculiaridades de las PFP es que ha sido común que el mismo resultado se interpretara de manera diferente en función del laboratorio en el que se realizaba el estudio, con amplias

\footnotetext{
* Autor para correspondencia.

Correo electrónico: JMartinezL@parcdesdalutmar.cat (J. Martínez Llorens).
}

discrepancias en lo que se definía como rango normal en una población sana ${ }^{6}$. La Global Lung function Iniciative (GLI) ha apostado, al igual que las sociedades científicas internacionales como la American Thoracic Society (ATS) y la European Respiratory Society (ERS), por ir cerrando el debate de cómo interpretar las PFP, especialmente con respecto al uso del límite inferior de normalidad y el $z$-score para diferenciar entre salud y enfermedad ${ }^{7,8}$. Recientemente la ATS ha recomendado también uniformizar la forma de expresión de los resultados de las PFP en el informe final, donde se deben incluir los valores absolutos y de referencia expresados como porcentajes de la media, el límite inferior de normalidad y el $z$-score e incorporar el grado de calidad de la prueba ${ }^{9}$. Además, la GLI ha uniformizado las ecuaciones de referencia que se pueden considerar como universales para parámetros de la espirometría en $2012^{7}$ y para la TLCO en $2017^{8}$. Las futuras iniciativas GLI incluirán ecuaciones de referencia tanto para volúmenes pulmonares estáticos como para el óxido nítrico exhalado y la FOT.

En la actualidad se utilizan herramientas para facilitar la comunicación entre los sistemas de salud propios de cada estructura sanitaria o centro con la finalidad de acceder a los datos de los pacientes; un ejemplo es la historia clínica electrónica (HCE). Para que los datos sanitarios del paciente puedan integrarse en la HCE deben ser estructurados y estandarizados. En este sentido, han surgido iniciativas con la espirometría forzada para integrar los datos en la HCE en Cataluña ${ }^{10}$ y en el País Vasco para programas de control de calidad centralizados ${ }^{11}$. Tener los datos estructurados también permite incorporar algoritmos de control de calidad que mejoren la cualificación de las maniobras realizadas durante la prueba de la espirometría ${ }^{12}$, minimizando su baja calidad en centros no especializados. Por último, es de destacar la reciente iniciativa liderada por la ATS de estandarizar los datos de las PFP en formato estructurado: posiblemente a principios de 2020 habrá un documento de consenso. Esto también facilitará la incorporación de las PFP en la HCE. Con estas iniciativas y mediante el big data analysis se podría 
producir nueva información valorable en cuanto a las relaciones entre parámetros de las PFP y los resultados de salud a lo largo de la vida que pudiera tener relevancia en el futuro.

Con la incorporación de las nuevas tecnologías ha habido también avances en el desarrollo de los equipos de función pulmonar más sencillos y sensibles. Existen espirómetros por ultrasonidos que no requieren calibración. La aplicación de estos espirómetros ultrasónicos a los sistemas de difusión ha permitido equipos más compactos y que además son capaces de medir la difusión de NO, diferenciando los componentes de membrana y capilar ${ }^{13}$. Nuevas tecnologías que utilizan las interrupciones de flujos son capaces de medir volúmenes pulmonares en sistemas compactos y portátiles que favorecerán su universalización. También se están incorporando progresivamente a nuestros laboratorios técnicas como la FOT y las pruebas de lavado de gases inertes de respiración múltiple. Sin embargo, seguimos siendo testigos de que exploraciones fundamentales en el diagnóstico de enfermedades respiratorias como la prueba de esfuerzo cardiopulmonar es identificada como exclusivamente cardiológica.

En estos últimos años las sociedades neumológicas nacionales e internacionales han apostado por la función pulmonar con programas de formación y rotación (Programas Aquiles Atenea, Ulises, ERS-SEPAR spirometry driving licence. EOS, etc. $)^{14,15}$. La formación reglada permitirá, sin lugar a dudas, un salto cualitativo en la calidad de los laboratorios de función pulmonar (LFP).

En este contexto de avances en el campo de las PFP, no parecía justificable que la acreditación de este tipo de unidades, auspiciada por la Sociedad Española de Neumología y Cirugía Torácica (SEPAR), no hubiera incluido a los LFP, hecho que está en vías de solución. Actualmente se están fijando los criterios de acreditación de los LFP en 3 diferentes unidades de alta complejidad, avanzadas y básicas, con opción de obtener el certificado de excelencia en cada nivel. En este tipo de acreditación de los LFP tendrá un papel muy relevante no solo la autodeclaración sino la evaluación que se está diseñando para cada uno de los niveles. La formación reglada y la acreditación de los LFP deben permitir un salto cualitativo en la calidad de las pruebas y un mayor conocimiento para de las nuevas generaciones de neumólogos.

En resumen, en los últimos años se está avanzado tanto en el aspecto tecnológico como en las indicaciones y en las normativas las PFP, sin embargo, no parecen llegar a los profesionales sanitarios que atienden a los enfermos con enfermedades respiratorias. Las sociedades científicas neumológicas nacionales e internacionales han apostado por potenciar la función pulmonar, aunque posiblemente deberían diseñarse estrategias para motivar a los profesionales en la realización y desarrollo de las PFP.

\section{Bibliografía}

1. Barratt S, Creamer A, Hayton C, Chaudhuri N. Idiopathic pulmonary fibrosis (IPF): An overview. J Clin Med. 2018;7:201.

2. Pirozzi CS, Gu T, Quibrera PM, Carretta EE, Han MLK, Murray S, et al. Heterogeneous burden of lung disease in smokers with borderline airflow obstruction. Respir Res. 2018;19:223.

3. Zimmermann SC, Tonga KO, Thamrin C. Dismantling airway disease with the use of new pulmonary function indices. Eur Respir Rev. 2019:28:180122.

4. Vasquez MM, Zhou M, Hu C, Martinez FDGS. Low lung function in young adult life is associated. Am J Respir Crit Care Med. 2017;195:1399-401.

5. Agustí A, Noell G, Brugada J, Faner R. Lung function in early adulthood and health in later life: a transgenerational cohort analysis. Lancet Respir Med. 2017;5:935-45.

6. Hall GL, Stanojevic S. The Global Lung Function Initiative (GLI) Network ERS Clinical Research Collaboration: How international collaboration can shape clinical practice. Eur Respir J. 2019;53:1802277.

7. Quanjer PH, Stanojevic S, Cole TJ, Baur X, Hall GL, Culver BH, et al. Multi-ethnic reference values for spirometry for the 3-95-yr age range: The global lung function 2012 equations. Eur Respir J. 2012;40:1324-43.

8. Graham BL, Brusasco V, Burgos F, Cooper BG, Jensen R, Kendrick A, et al. 2017 ERS/ATS standards for single-breath carbon monoxide uptake in the lung. Eur Respir J. 2017:49:1-31.

9. Culver BH, Graham BL, Coates AL, Wanger J, Berry CE, Clarke PK, et al Recommendations for a standardized pulmonary function report. An officia American Thoracic Society technical statement. Am J Respir Crit Care Med. 2017:196:1463-72

10. Salas T, Rubies C, Gallego C, Muñoz P, Burgos F, Escarrabill J. Requerimientos técnicos de los espirómetros en la estrategia para garantizar el acceso a una espirometría de calidad. Arch Bronconeumol. 2011:47:466-9.

11. Marina Malanda N, López de Santa María E, Gutiérrez A, Bayón JC, Garcia L, Gáldiz $\mathrm{JB}$. Telemedicine spirometry training and quality assurance program in primary care centers of a public health system. Telemed e-Health. 2014;20:388-92.

12. Burgos F, Melia U, Vallverdú M, Velickovski F, Lluch-Ariet M, Caminal P, et al Clinical decision support system to enhance quality control of spirometry using information and communication technologies. JMIR Med Informatics. 2014;2:e29.

13. Puente Maestú L, de Miguel Diez J, López Padilla D. Prueba de difusión por respiración única. La longevidad es la recompensa de la virtud. Arch Bronconeumol. 2018;54:501-2.

14. Brightling C, Genton C, Bill W, Welte T, Gaga M, Heuvelin E, et al. ERS Clinical Research Collaborations: Underpinning research excellence. Eur Respir J. 2018;52, 1801534.

15. Programa de formación en Espirometría SEPAR-ERS [consultado 1 octubre 2019]. Disponible en: https://www.separ.es/?q= node/1467. 